
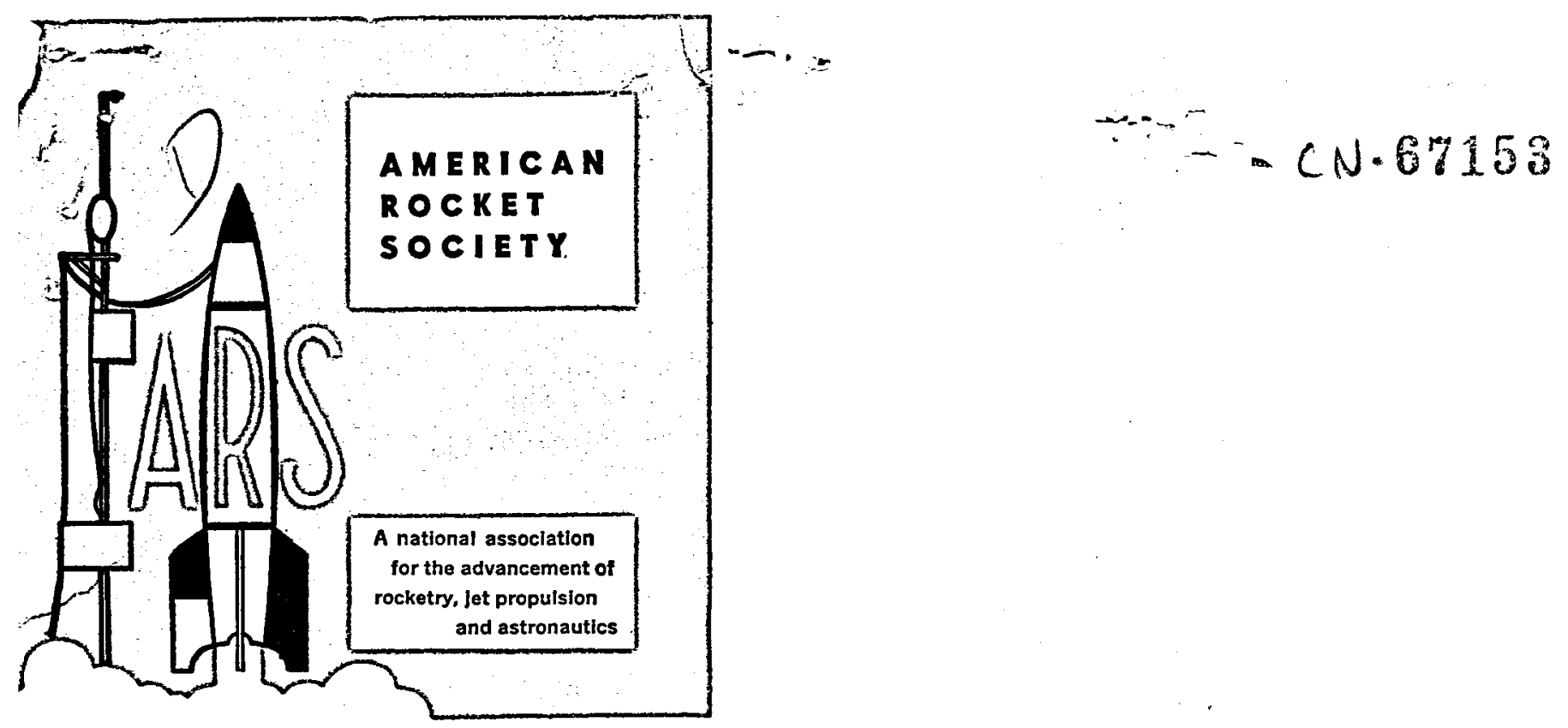

500 FIFTH AVENUE - NEW YORK 36, N. Y.

Publication No. 145

THE EXPLORER

ROCKET RESEARCH PROGRAM

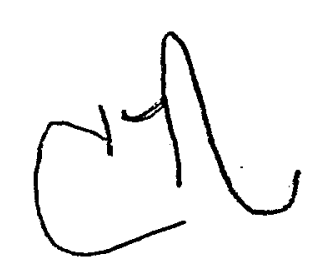

1

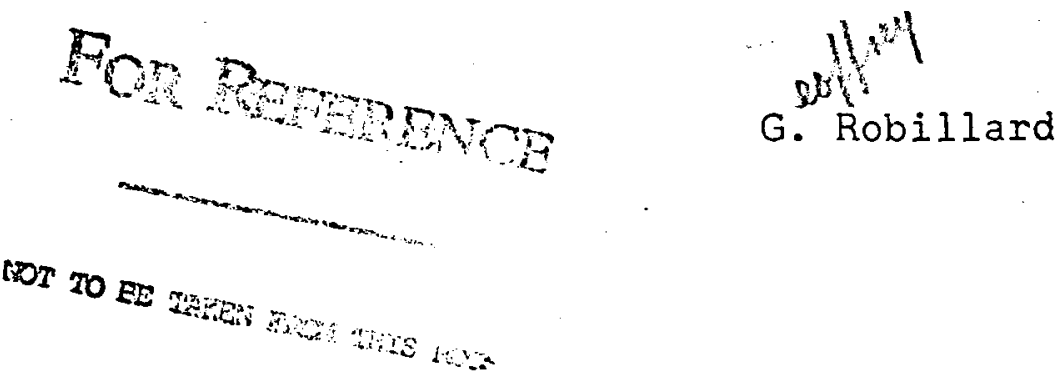

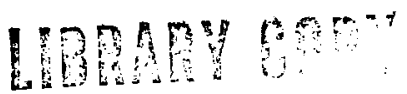

DEC 311903

LAiviter RESE

LIBRGRi, KGE

LANGLEY FIELD, VIRGINIA

Presented at the ARS 13th Annual Meeting, Hotel Statler, New York, New York, November 17-21, 1958

Publishing rights reserved by the American Rocket Society. Abstracts may be published wi thout permission if credit is given to the author $718-58$ and to ARS

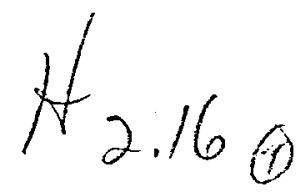




-




\section{ORDCIT Project}

Contract No. DA-04-495-Ord 18

Department of the Army

ORDNANCE CORPS

Publication No. 145

THE EXPIORER

ROCKET RESEARCH PROGRAM

G. Robillard

Copy No.

JET PROPULSION LABORATORY

California Institute of Technology

Pasadena 3, California

October 31, 1958

a 1283994 


\section{INTRODUCTION}

Since September of 1956, nine Jupiter-C missiles have been launched from the firing pad at Cape Canaveral. The first Jupiter-C firing tested the propulsion system, air frame, and guidance components of the missile, and the second and third firings tested a model of the Jupiter nose cone under realistic re-entry conditions. The remaining six Jupiter-C missiles were used as the launching vehicles for EXPLORER satellites I through VI (Fig. 1). Of the six satellite firings, EXPLORERs I, III, and IV achieved satisfactory orbits.

The Jupiter-C missile was designed and developed as a joint program under the technical direction of the Jet Propulsion Laboratory and the Army Ballistic Missile Agency. The Jet Propulsion Laboratory developed the three high-speed stages, and the Army Ballistic Missile Agency handled the development, construction, and operation of the first-stage booster rocket and the guidance system. Many other organizations have contributed to the success of the EXPLORER satellite program, most notably the State University of Iowa, the Air Force Cambridge Research Center, and the satelite tracking teams of the Variguard organization. 


\section{DESIGN OF THE JUPITER-C}

The Jupiter-C missile is a four-stage vehicle. The first stage consists of a modified Redstone rocket. The three high-speed stages are comprised of clusters of solid-propellant rockets placed in a spinning tub which is mounted in the nose of the first stage.

The standard Redstone missile delivers a thrust of 75,000 pounds and employs alcohol as the fuel and liquid oxygen as the oxidizer. For the EXPLORER missions, the propellant tanks were enlarged in order to permit an increase in burning time to 155 seconds, and the alcohol was replaced by another fuel, called hydyne. This latter change resulted in an increase in thrust of about 8000 pounds giving a total thrust of 83,000 lbs. The fuel change was effected without major modification of the engine.

The last three stages of the missile are spun in order to minimize dispersion of the payload due to any thrust malalignment of the unguided solid-propellant rocket clusters. The tub is spun by two battery-powered electric motors mounted in the top of the instrument compartment, which is emplaced between the tanks of the first-stage rocket and the spinning tub and contains the guidance and control equipment for the missile. This compartment also holds a spatial attitude control system for horizontal alignment of the high-speed stages before the ignition of the second stage.

The second stage of the Jupiter-C is composed of an annular ring of eleven solid-propellant motors (Fig. 2), each of which is a 6-in.-D scale model of an early version of the powerplant of the SERGEANT missile. All of the solid-propellant motors of the three 
high-speed stages are identical in configuration. This motor was chosen for this task because it was a reasonably high-performance rocket and was known to be extremely reliable, having undergone over 300 static tests. The eleven-motor ring of the second stage is mounted in bulkheads inside the spinning tub and is held accurately in position by launching lugs. Extremely accurate positioning and balance of the three upper stages and the payload are required in order to prevent vibration caused by the spinning tub and to minimize dispersion of the stages in launching and firing. Because of the centrifugal forces on the spinning second-stage motors, it was considered necessary to test these motors under simulated flight conditions on the ground. The radial load amounts to $180 \mathrm{~g}$ at the full spin rate of $700 \mathrm{rpm}$. Pairs of motors were spun and fired in a static-test jig. The motors operated well, but it was necessary to make a small change in the nozzle design in order to prevent the cantilevered nozzles from deflecting outward under the spin loads.

The third stage (Fig. 3) consists of three motors identical to those in the second stage, which are connected by bulkheads in a fashion similar to that used for the second stage installation. The three-motor cluster fits inside of the ring of motors comprising the second stage and is held in place by launching lugs.

A conical structure attached to the top of the third stage contains the timer and batteries for firing the third and fourth stages and serves to support the single fourth-stage motor. This motor is identical in configuration to those of the other high-speed stages, except in the manner in which it is supported and in the fact 
that the nozzle exit diameter is somewhat smaller to allow adequate launching clearance.

By the time permission was obtained to fire EXPLORER I, sufficient tests had been made of a propellant having improved performance over that used in SERGEANT to permit its use in the fourth-stage motor. The fourth-stage rocket places the lowest requirement on the physical properties of propellant in that it is spun on its own axis; hence the radial loads are a minimum. Previous to the firing of EXPLORERS IV AND $V$, this propellant was qualified by extensive static spin-testing for use in the third stage, and an even more energetic propellant had qualified for use in the fourth stage. These propellant changes, together with a decrease in the weight of some of the inert parts by design refinements, account for the increased payload weights of the latter satellites. Some potential for increased payload using this missile configuration still exists, but the return in terms of payload weight is rapidly diminishing with respect to the amount of development effort required.

The satellite payload (Fig. 4) is rigidly attached to the head end of the fourth-stage rocket motor through an insulating ring which forms the antenna gap for one of the two payload transmitters. The second transmitter operates across a similar gap between the cylindrical portion of the payload and its nose cone.

The entire high-speed assembly, including the payload, is very carefully aligned and balanced in its tub before the assembly is mounted on the Redstone booster.

The design of the Jupiter-C was carried out to afford a vehicle which would provide maximum reliability with minimum development 
effort. For this reason, proven components were used wherever possible, and in order to assure reliability redundant systems were used in many places to prevent system failure caused by malfunction. The ignition system for the high-speed stages is one example of such redundancy. The problem of reliable ignition at high altitude was resolved in three ways: (1) An igniter was designed which would fire the motors reliably in a laboratory vacuum. (2) The rocket motors were sealed so that they would hold atmospheric pressure. (3) The igniter was sealed in an inner container capable of holding atmospheric pressure if the motor seal should leak. 


\section{FIRING SEQUENCE}

Prior to takeoff the tub is spun up to $550 \mathrm{rpm}$ and held at this speed. About 70 seconds after takeoff, a governor controlled by the missile programmer increases the rotational speed gradually up to $750 \mathrm{rpm}$ at a time about 20 seconds before first-stage burnout. This procedure eliminates resonance between the spin frequency and the natural bending frequencies of the missile. These bending frequencies increase as the propellants of the first stage are consumed; thus the spin rate is increased gradually so that the spin frequency always remains between those of two of the natural modes of vibration of the missile.

The missile takes off vertically under its thrust of 83,000 pounds. During the 155-second burning time of the first stage, the vehicle is tilted into a trajectory which is approximately 40 degrees inclined to the horizon at burnout. A depletion cutoff technique is used to maximize the impulse delivered by the Redstone motor. Contacts are placed in the discharge lines from the fuel and oxygen pumps; whichever of these contacts first senses a pressure drop triggers a relay which closes both propellant main valves to the combustion chamber. Five seconds after burnout a timer activates the six explosive bolts which secure the guidance compartment to the tank section of the first-stage motor. Coil springs wrapped around these bolts exert a gentle push on the guidance compartment and separate it cleanly from the booster. The failure of EXPLORER $V$ to achieve orbit is attributed to a malfunction at this point in the launching sequence. In this one instance, the Redstone booster was still 
exerting some thrust after separation; hence the separated booster section was able to overtake and ram the guidance compartment. This impact caused the gyros in the guidance compartment to tumble; consequently, the reference planes were misoriented during the ensuing portions of the launching sequence.

From the point of separation, the two portions of the missile coast through a vacuum trajectory until the apex is attained after about 404 seconds after takeoff. During the free-coasting period between separation and apex, the spatial attitude control system in the guidance compartment aligns the high-speed stages into an exactly parallel position with respect to the local horizon. This reorientation uses the gyros of the guidance compartment as reference and is accomplished by means of small compressed-air nozzles located at the base of the guidance compartment.

Because of the relatively crude cutoff technique based on propellant depletion used in the first stage, it is impossible to predict the exact time when apex will be attained. Only by igniting the highspeed stages at the moment of apex and by accurate alignment of the stages in the horizontal direction can orbital flight be assured. Three independent methods are employed to determine the time of apex: first, a radar tracking plot is used to determine the time at which apex will be reached; second, an accelerometer in the missile relays by telemetry the buildup of velocity in the first stage; and third, standard Doppler tracking furnishes the same information. The data obtained from these three sources are fed into a small computer that provides an average estimate of apex time, but allows for the respective quality of the three types of data. This value is then 
used to set a timing device which sends a radio signal to the missile to fire the second stage. The computation is accomplished in the 4-minute interval between separation and apex.

Each of the three high-speed stages has a burning time of about 6 seconds, and 2 seconds of coasting time are allowed between the firing of successive stages to permit complete thrust decay. The second-stage firing signal is received by the command transmitter in the instrument compartment, and the stage is fired by an electrical signal from batteries in the guidance compartment. The pressure rise in the second-stage motors signals the start of a timer housed in the conical support for the fourth stage, which 8 seconds later fires the third-stage and, 8 seconds after that, the fourth stage. As each stage fires, it breaks shear pins holding it to the preceding assembly and leaves that stage behind to fall back to earth.

The failure of EXPLORER II to achieve an orbit has been traced to a structural failure in the igniter support for the fourth stage. The light plastic cone which supported the igniter from the nozzle end of the rocket motor apparently failed under the combined spin, inertial, and vibration loads, thereby allowing the igniter to slip out through the nozzle into the conical structure supporting the fourth stage. Strengthening the igniter support prevented this malfunction in subsequent rounds.

The payload of EXPLORER VI contained a fifth small solidpropellant rocket motor stage oriented in such a manner that its nozzle exhausted through the nose cone of the payload. This motor was designed to be fired by a timer at apogee after the first halfrevolution of the satellite around the earth. At this time the motor 
would have been oriented in such a way that it could impart additional velocity to the payload along its path, thereby raising the perigee height well above its initial value. This "kick-in-the-apogee" technique is an economical way of achieving a high orbit because it eliminates the necessity of raising all of the missile hardware to a high altitude. 


\section{PAYIOADS AND ORBITS}

The entire orbiting assembly in EXPLORER $I$, that is, the empty rocket motor shell from the fourth stage plus the instrument compartment, weighs $30.8 \mathrm{lbs}$. The instrument compartment alone weighs 18.0 lbs. The total assembly is 80 inches long and 6 inches in diameter. This satellite achieved an initial orbit having a perigee altitude of 225 miles and an apogee altitude of 1594 miles, corresponding to a period of revolution of 114.78 minutes. Tracking data showed that the satellite was injected into orbit at an angle only about 0.8 degrees from the horizontal, although a suitable orbit would have been achieved even if this error were as great as 4 degrees.

The shell of the instrument compartment was constructed of 410 stainless steel, 0.025 inches thick. The outside surface was sandblasted and then coated in stripes with aluminum oxide (Rokide A). The region of the stagnation point of the nose cone was completely covered to guard against aerodynamic heating during launch. The payload surface was treated in this manner so that the heat absorbed by radiation from the sun and by reflected radiation from the earth plus the heat generated internal to the payload would balance the heat radiated by the payload to space. By this means, it was possible to maintain the electronic components within a temperature range in which they could function properly. Because this technique could not be expected to maintain a constant temperature of the satellite skin as the satellite moved into and out of the earth's shadow, the sensitive components were thermally insulated from the skin to smooth out the temperature fluctuations. The success of this technique is 
indicated by the fact that the maximum and minimum temperatures recorded within the instrument compartment were 43 and $104^{\circ} \mathrm{F}$, respectively.

EXPLORER I carried two minimal-weight transistorized ratio transmitters. The low-power transmitter radiated 10 milliwatts of energy, whereas the second transmitter radiated 60 milliwatts. Because of its higher power requirement, the 60-milliwatt unit transmitted for only about 2 weeks; however, the low-power transmitter was tracked for several months after launching.

The primary mission of the transmitters was to provide a signal for tracking the satellite. The high-power transmitter could be received by sensitive amateur receivers, but the low-power transmitter could be tracked only by the more sophisticated narrow-band Microlock and Minitrack receivers. In addition to providing a tracking signal, the transmitters were used to telemeter scientific data from the satellite; this data included measurements of temperature, micrometeorite impacts, and cosmic-ray intensity.

Temperatures were measured at four points: one directly under the stagnation point of the nose cone, two others at two locations on the outer skin, and another directly within the electronic package itself.

Micrometeorite impacts were counted by two devices. A sensitive microphone attached to the inside of the payload shell, by means of an amplifier and a scale-of-two circuit, caused a frequency shift of a subcarrier oscillator each time an impact occurred anywhere on the satellite. In addition to the microphone, a micrometeorite erosion gauge consisting of eleven grids of fine brittle wire was mounted 
just forward of the nozzle of the last-stage rocket motor. These grids were wired in parallel; each time a grid was broken by micrometeorite impact the resistance of the circuit changed, and the change was reflected in the frequency of a subcarrier oscillator.

The intensity of cosmic radiation was detected by a GeigerMueller tube. This instrument was able to count only the number of impingements within its sensitivity range and was unable to differentiate between particles of various energy levels. Inasmuch as the cosmic-ray intensity had been predicted to be of the order of 30 to 40 counts per second, the range of the instrumentation was set to bracket this range. The field of cosmic radiation encountered at altitudes greater than 600 miles, however, was so intense as to saturate the instrument completely. The only conclusion which could be reached was that the intensity was at least 1000 times greater than expected.

With EXPLORER I, telemetering data could be gathered only when the satellite was over one of the ground-receiving stations. These stations were located in Florida, Nigeria, Singapore, and California, and at a number of places along the $65^{\circ}$ west meridian. Because of the relatively small number of receiving stations, much of the orbit was not observed, and for this reason much of the telemetered data was lost. In order to eliminate this difficulty, EXPLORER III carried a miniaturized tape recorder which stored the information acquired throughout one complete orbit and reported it on command when the satellite passed over a suitably equipped receiving station. Playback was accomplished at high speed so that approximately 2 hour's data could be transmitted in 5 seconds. Between interrogations the 
high-power transmitter was turned off to conserve power. Only the cosmic-ray information was transmitted in this fashion; the other experiments, similar to those in EXPLORER $I$, were telemetered continuously by the low-power transmitter.

EXPLORER III attained an orbit having a perigee altitude of 120 miles and an apogee altitude of 1740 miles, with a period of 115.8 minutes. The orbit was inclined about $331 / 2$ degrees to the equator. The greater ellipticity of this orbit was caused by a significant elevation exror at injection. In retrospect, this represents a particularly favorable malfunction since the high-apogee orbit has made it possible to obtain exceedingly interesting data from the cosmic-ray experiment.

The instrumentation in EXPLORER IV was designed specifically to study the cosmic radiation in greater detail. Improvements in the performance of the high-speed stages for this firing permitted the payload weight to be increased by 7 pounds. By eliminating all of the other experiments, including the tape recorder, it was possible to package four instruments for measuring cosmic-ray data in this satellite. Two scintillation counters were carried in addition to two Geiger-Mueller tubes similar to those carried in the earlier satellites. One of the Geiger tubes is shielded with lead to eliminate data below fixed energy levels. The other tube is set to respond to any particle which penetrates the shell of the satellite and the casing of the Geiger tube. One of the scintillation counters records a pulse each time a particle passes through it. The other can measure the energy level of the particles which it encounters. Both scintillation counters are shielded by lead, except for one 
window in each where the shielding has been made as light as possible and a hole has been cut in the shell of the satellite.

EXPLORER IV was fired on a much more northerly orbit than its predecessors. It achieved a perigee altitude of 160 miles and an apogee altitude of 1390 miles; the period of the satellite was 110.3 minutes. Its orbit inclined 50.4 degrees to the equator. By this means, it was able to sample a far greater volume of space with respect both to latitude and to altitude than were the earlier vehicles. 


\section{SCIENTIFIC DATA}

The justification for a project of this magnitude, of course, is the scientific data which are obtained. The EXPLORER project has, in fact, acquired a great deal of very interesting data; so much so that it may be several years before this extensive accumulation is completely analyzed. However, sufficient preliminary information is now available to sketch a rough picture of space in the vicinity of the Earth.

Although the temperature measurements taken inside the satellite were not intended to measure any fundamental quality of the space environment, they served to prove the success of the scheme which had been employed to control the satellite's temperature. We now have demonstrated that, at least insofar as temperature is concerned, we shall be able to provide a habitable environment for man in space.

The rate of decay of the orbit, particularly that of EXPLORER III, which lasted in orbit for about 3 months, has provided a measure of the density of the upper regions of the atmosphere. EXPLORER III completed approximately 1260 revolutions before its disappearance. Its orbit ranged from a perigee of 116.86 miles and an apogee of 1740.77 miles on its first pass to 107.2 miles and 650.2 miles respectively about 2 weeks before it vanished. The rate of decay of apogee was $11.36 \mathrm{miles} /$ day between March 26 (the date it was launched) and April 11, 11.95 miles/day between April 11 and 25, $14.99 \mathrm{miles} /$ day between April 25 and May 31, 14.16 miles/day from then until June 10, 
and $16.9 \mathrm{miles} /$ day thereafter. The exact significance of this somewhat erratic path in terms of atmospheric density is still being analyzed.

The measurements of micrometeorite density have substantially confirmed previous estimates based on such indirect evidence as the data from high-altitude sounding rockets, and the measurement of ocean-floor sediment. The results of a partial analysis of the data from EXPLORER I by the Air Force Cambridge Research Center, which is responsible for this experiment, show for example that the average flow of particles in the 4-micron range is 30 particles/sq $\mathrm{yd} / \mathrm{hr}$, whereas for a larger particle of about 10 microns in diameter the rate is 3 particles/sq $y d / h r$. For the space-ship designer, these numbers offer no problem. However, they take on an interesting meaning when the surface considered is the surface of the earth. In these terms, we find that cosmic dust accounts for an increase in the mass of the earth of 2000 tons per year.

It is interesting to note that the Russians carried on a similar experiment aboard the Sputniks. Surprisingly, they have reported a density of particles which is approximately 1000 times as great as that measured by EXPLORER. This result is difficult to understand not only because it disagrees with the previous estimates, but also because the increase in mass of the earth at this high rate would be expected to have had a noticeable effect on its speed of rotation over the centuries.

In contrast to the micrometeorite measurements which appear to verify preliminary estimates, the measurements of cosmic-ray intensity gave some surprising new results. The first two EXPLORERs indicated 
that the level of cosmic radiation was about as expected up to 600 miles altitude in the region near the equator. From this altitude up to the highest altitude reached by the sateliites, the cosmic-ray intensity increased at a much higher rate than had been expected. At the farthest reaches of the orbits, the count was more than 1000 times the expected value, and the rate of increase showed no tendency to level off.

Preliminary analysis of the data from EXPLORER IV shows that the number of cosmic-ray particles passing through the unshielded. Geiger tube is of the order of $36,000 / \mathrm{sq} \mathrm{cm} / \mathrm{sec}$, from all directions. At least $60 \%$ of these particles have enough energy to penetrate the lead shield around the other Geiger tube. In addition to these very energetic particles, there is an even greater density of particles of low energy. At the northern and southern limits of the trajectory, these low-energy particles predominate. Here, the flux of particles of low energy at an altitude of about 1400 miles is about one million/ $\mathrm{sq} \mathrm{cm} / \mathrm{sec}$, from all directions. As the satellite rotates around its axis, the density measurements change. On the basis of this result, it appears that more than $80 \%$ of these particles come from one favored direction.

On the basis of the preliminary estimates, it is not possible to separate altitude effects from latitude effects, and, because of the magnetic field, both of these effects should cause changes in cosmicray intensity. The first 2 week's data indicated an increasing intensity with altitude at all latitudes. The radiation has been found to vary both in quantity and in quality with latitude and altitude. A low-energy component similar to that suggested on the 
basis of the results of the first two EXPIORER satellites is usually present, whereas high-energy particles become more and more predominant as the satellite approaches the magnetic equator. Thus radiation intensities in excess of 100 Roentgens/hr have been observed by the satellites.

In regard to cosmic radiation, it is interesting to note that, although the Russian satellites also carried instruments for measuring this effect, the preliminary announcements of scientific results made by the Russian scientists did not indicate that they had discovered this high-intensity region. Their preliminary information was confined to altitudes below 600 miles. At a recent meeting sponsored by the International Geophysical Year Committee held in Moscow, the Russians entered into a more thorough description of the equipment on board the Sputniks. They revealed that the Sputniks carried tape recorders which stored scientific information around the orbit and played it back as the Sputnik passed over Russia. Also, the Sputniks carried continuous transmitters which sent down the information to earth steadily for any receiver which the satellite happened to be passing over. The cosmic-ray measurements were being transmitted on the continuous transmitter in the same way that information is telemetered from EXPLORER's I and IV, but the cosmic-ray information was evidently not put on the tape recorders as was done with EXPLORER III. Thus the Russians could observe cosmic ray data only when the satellite was near its lowest point. When the satellite was passing through the high-altitude region, and presumably detecting the intense cosmic-ray field, the satellite was not over Russia. Instead, when 
the satellite was passing through this interesting region, it was over some part of the world near or below the equator.

- It is true that many radio-receiving stations over these portions of the world were recording the signals sent down by the Russian Sputniks. However, these signals were meaningless without the telemetering code, and this code was not published by the Russians. Furthermore, in spite of the request by this country and others, the Russians would not enter into any arrangements to exchange preliminary satellite data. Thus, although the Sputnik was observing this surprising high-intensity cosmic-ray field, the Soviet government, by its own decision, refused to accept this data or make it possible for anyone else to interpret it. As a result, although the soviet scientists were the first to launch an artificial earth satellite, they were the last to learn about this vital discovery of the highintensity cosmic-radiation field. Instead, it remained for the American scientists to discover and announce this completely new geophysical phenomena.

The Jupiter-C research vehicle then, however unsophisticated it may seem as compared with Vanguard, and however diminutive it may be as compared with Sputnik, has been to date exceedingly successful in accomplishing its objective: the exploration of space and the acquisition of fundamental knowledge of our planet's environment in the universe. 


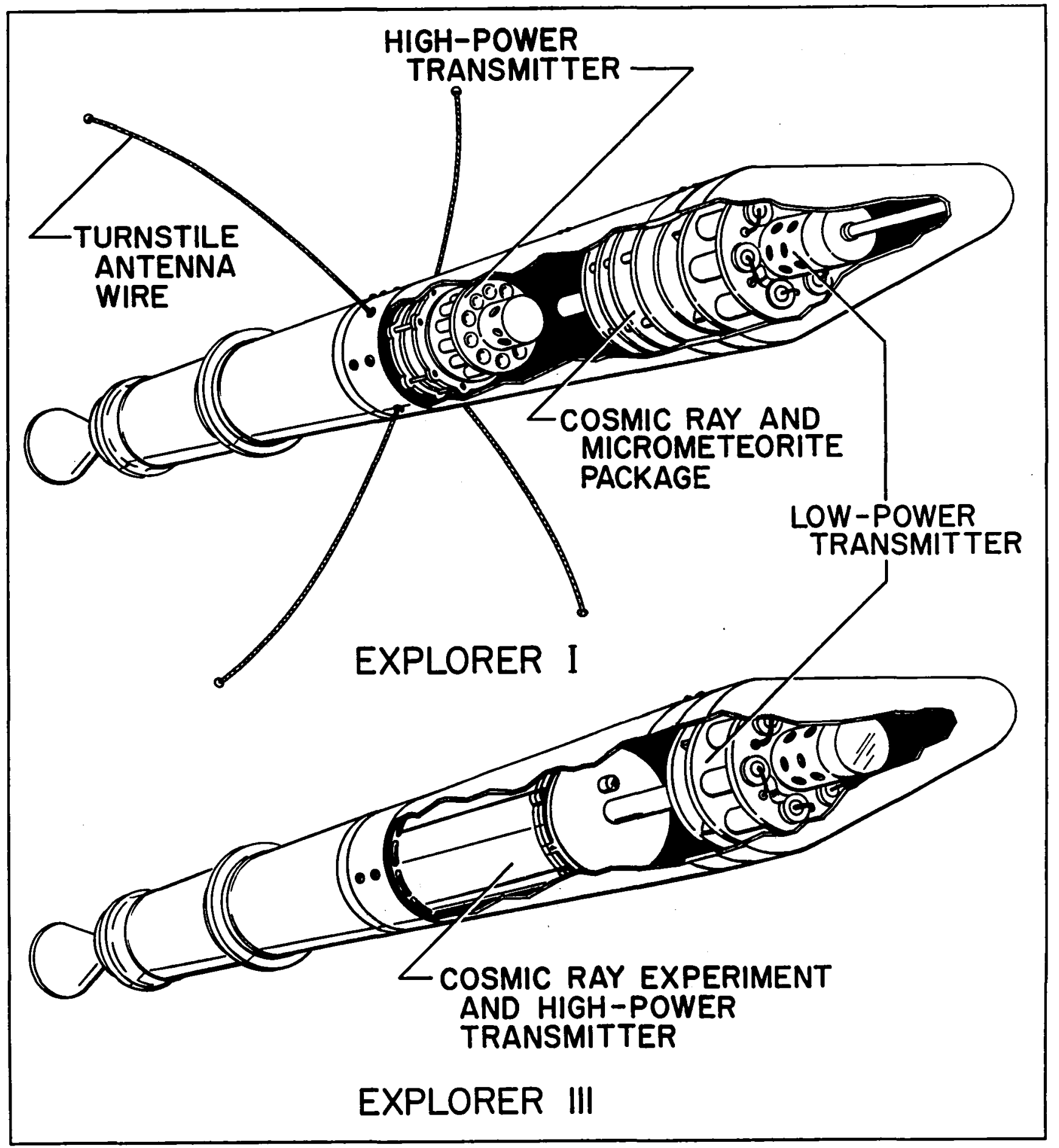

Fig. 1. Cutaway Views: EXPLORER I and EXPLORER III 


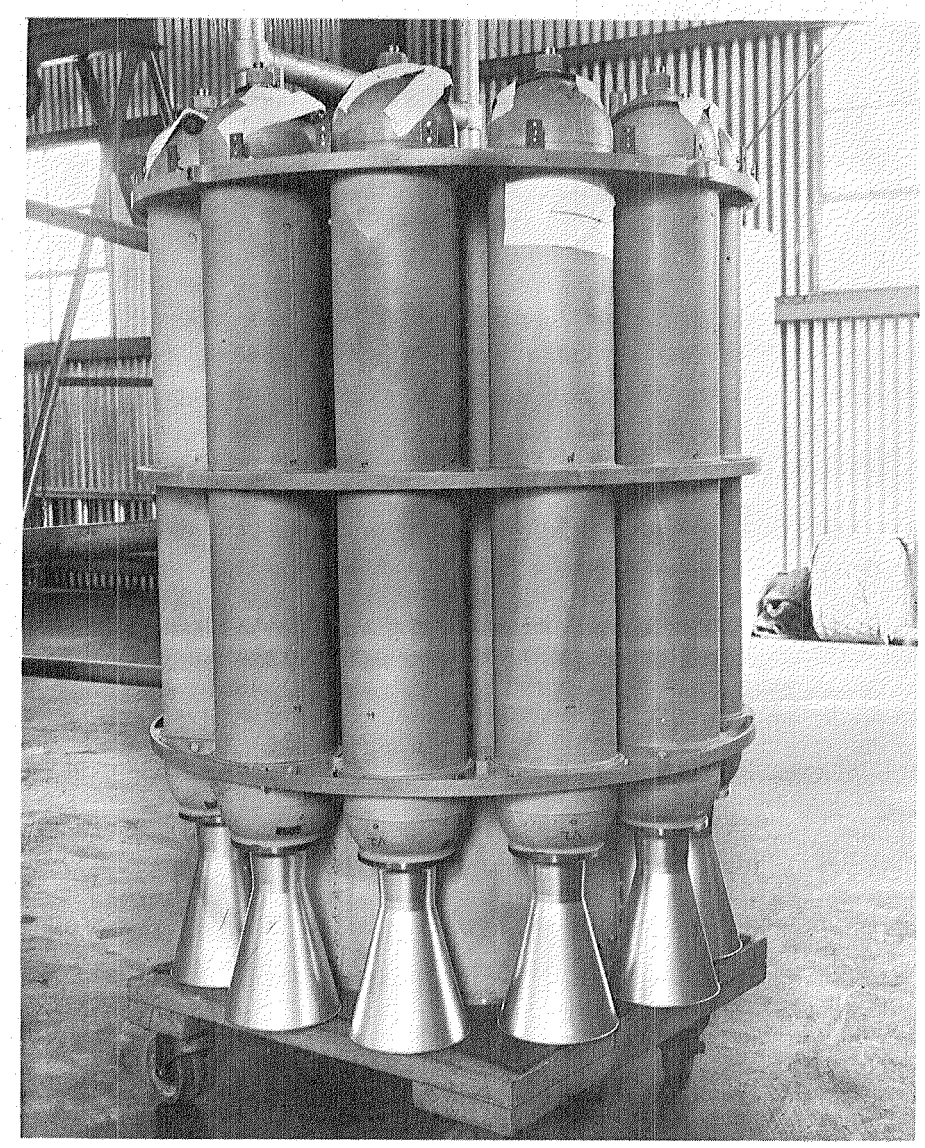

Fig. 2. Second-Stage Cluster of Solid-Propellant Motors

Fig. 3. Third-Stage Motor Cluster

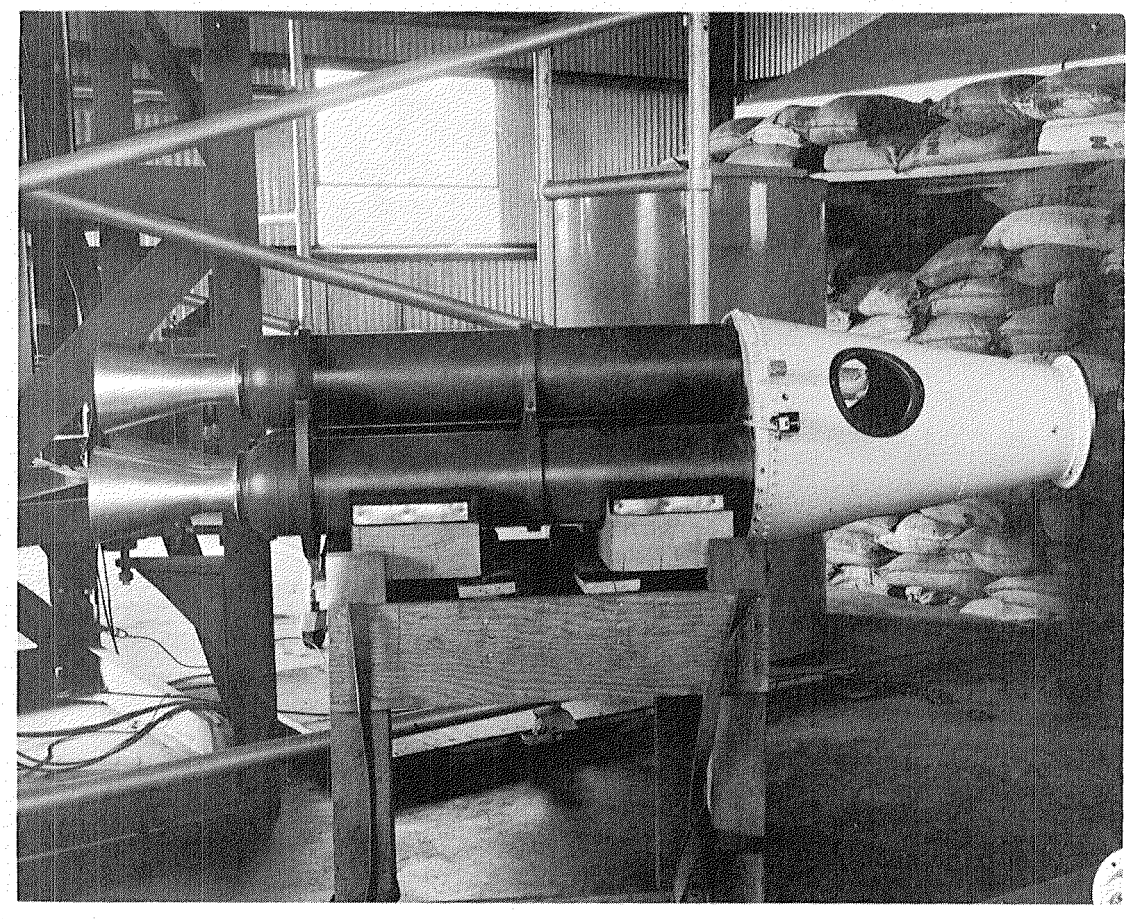

Page 21 


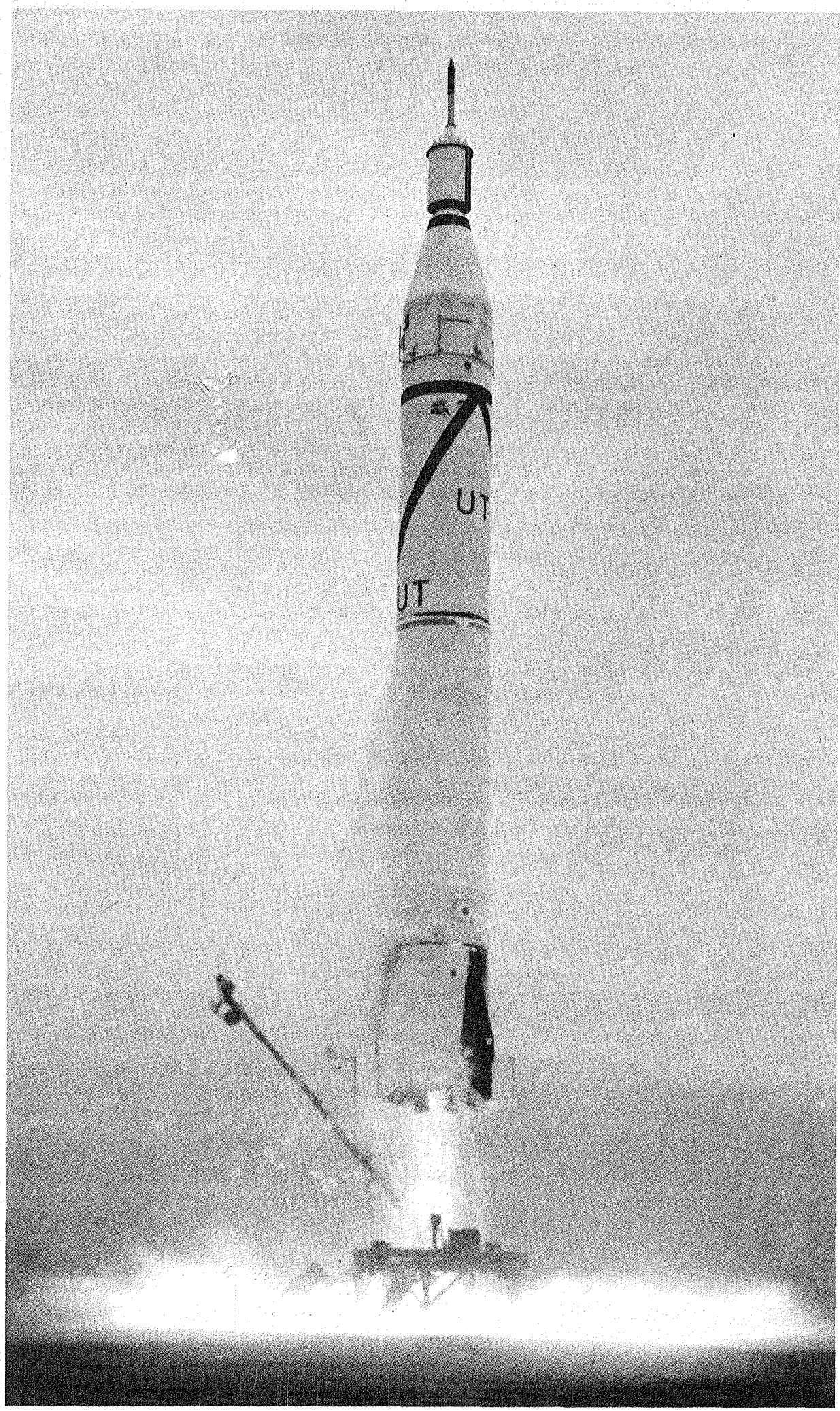

Fig. 4. EXPLORER Launching 
End of Document 